\title{
A simple methodology to improve AIS-based assessment of recovery after acute spinal cord injury
}

Spinal Cord (2005) 43, 61-63. doi:10.1038/sj.sc.3101665; Published online 2 November 2004

Recently, Curt et al ${ }^{1}$ summarized in Spinal Cord nicely the requirement for precise quantitative and qualitative neurophysiological assessment of functional recovery after spinal cord injury (SCI), pioneering a novel clinical basis for the implementation of interventional therapies. This is strongly needed as, to date there is a lack of valid evidence (class I) of an effective pharmaceutical treatment following acute SCI (meta-analysis) that meets the requirements of the Cochrane collaboration. ${ }^{2}$

The lack of therapeutic options also refers to several immanent methodological difficulties in the assessment of therapeutic effects. In general, difficulties conducting SCI trials during the acute phase are due to the rather low incidence of SCI and heterogeneity of the lesion (level, extension, bleeding) and patient (age, sex, comorbidity, medication). Thus, it appears that high patient numbers might be one key for better data acquisition, even though continuously increasing the number of patients culminating in 800 (Figure 1) recent trials did not result in significant improvements. Owing to the rather low incidence of SCI, this leads to prolonged recruiting periods. In addition, score results can not be directly linked to the initial neurological status, distribution and intensity of neurological deficits. ${ }^{3}$ In 2001, for the first time, the authors of the trial investigating effects of GM-1 demonstrated the heterogeneity of data using (changed) summed scores in detail. They fail to obtain homogenous, comparative groups even following stratification by means of ASIA. ${ }^{4}$ This leads to difficulties in correlating statistical results to neurological recovery challenging the solely widespread use of classifying subpopulations according to $\mathrm{AIS}^{5}$ in SCI trials.

In order to save resources, we propose a simple, AIS-based method aimed at facilitating improved assessment of recovery after SCI. In detail, for future SCI trials, we propose the alternative use of absolute (linear) numbers (dermatomes or myotomes revealing significant recovery: $>2$ points improvement) instead of changed summed (ordinal) scores that are at risk of overpronouncing nonrelevant changes subjective to interinvestigational differences. Conventional AIS procurement just summarizing sensory and motor points for different dermatomes/myotomes (summed score result, nonlinear), is complicated by the fact that small nonsignificant differences add up - therefore creating false positives, indicating statistically significant recovery (Figure 2). This problem can be mathematically overcome by using the difference of two examinations to detect significant signs of significant recovery (absolute, linear). In brief, we propose significant motor recovery for one myotome to occur by the increase of two or more motor points analyzed according to the ASIA-Scale between two examination time points. We suggest this ' $>2$ points improvement' as a lucid indicator to reflect the clinical neurological improvement of the individual patient (eg improvement from total palsy to active movement without gravity; flicker of contraction to active movement with gravity). A ' $>2$ points improvement' might minimize nonrandom chance effects (ie by interexamination and interinvestigator variations, Figure 2) leading to a higher risk of detecting false-positive or -negative therapeutic effects caused by 'one-point changes-differences' in multiple myotomes.

The difference between two examinations of myotome numbers with ' $>2$ points improvement' as indicator for

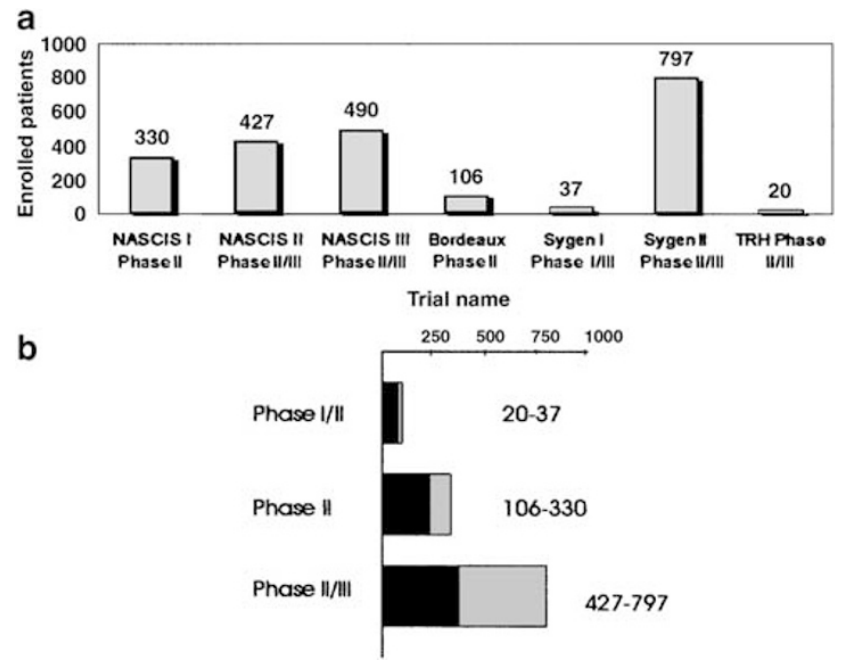

Figure 1 Many patients enrolled in randomized controlled trials in acute nonpenetrating SCI fail to deliver class I evidence of efficacy (Cochrane collaboration). In detail, Phase I/II trials (toxicity/efficacy) span from 20 to 37 patients, Phase II trials (efficacy) from 106 to 330 patients and Phase II/III (efficacy) from 427 to 797 patients. Dark bars represent the minimum numbers and light gray the maximum numbers of enrolled SCI patients 

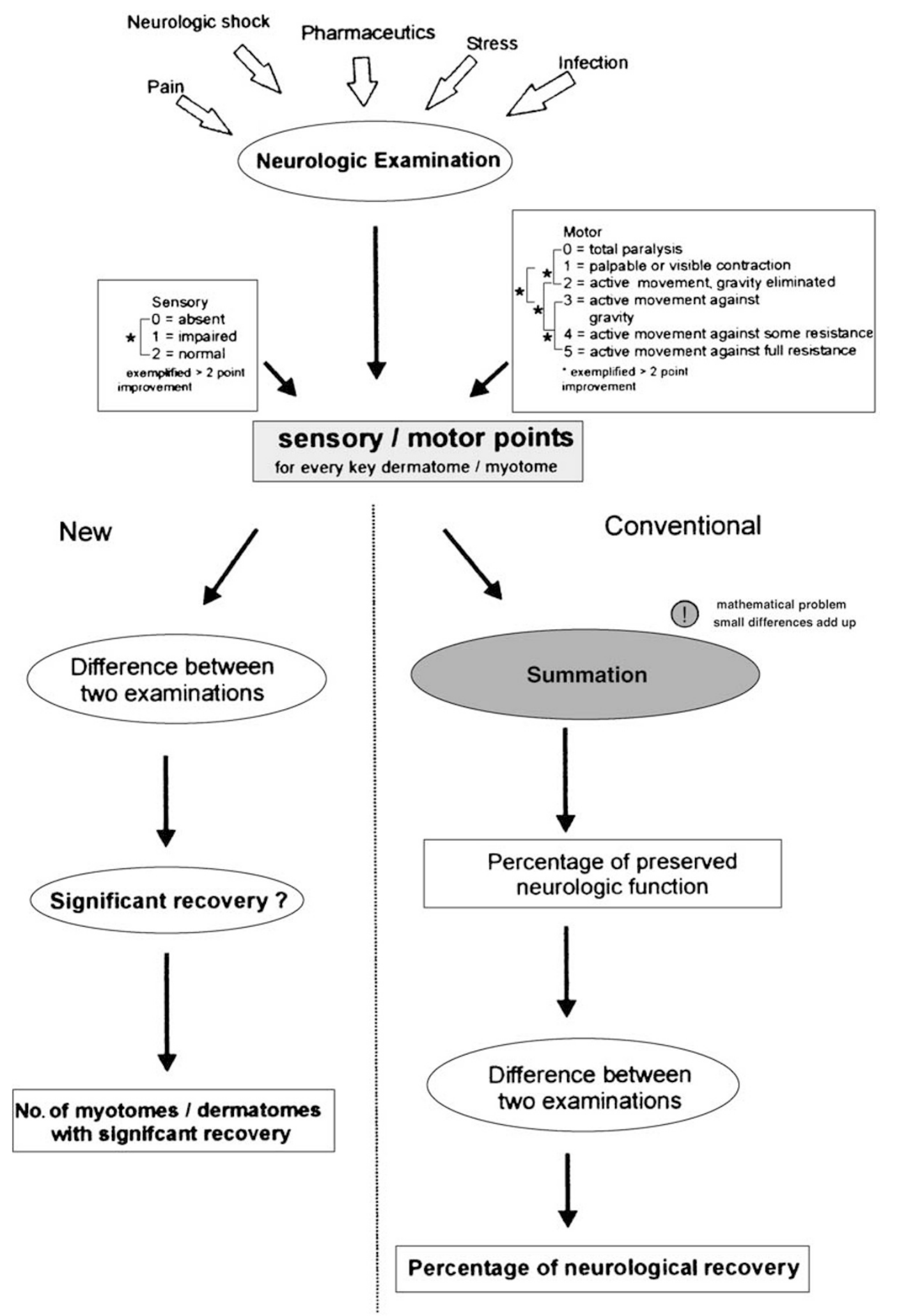

Figure 2 Proposed simple alternative for the use of changed summed (ordinal) scores (right side) suggesting the use of absolute (linear) numbers of dermatomes or myotomes revealing significant recovery (' $>2$ points improvement') to enhance the comparability of individual recovery patterns (left side). Since data recruitment is identical to the ASIA basis, conventional and new data procurement can be performed in parallel without additional requirements or risk of loss of conventional data 
significant recovery would be functionally more meaningful to the individual patient than the use of changed summed scores. Importantly, linear data procurement might enhance statistical power, thereby minimizing the number of patients needed to detect therapeutic effects. Furthermore, this method might be of help to enhance comparability of individual recovery patterns.

A precise predictive heterotypization is essential for effective stratification of acute SCI patients and a prerequisite to detect presence or absence of statistically significant recovery in future SCI trials. ${ }^{1,4,6}$ A pioneering collaborative consort formation of several highly specialized spinal cord centers was formed to pinpoint enough adequate comparable, eligible SCI patients (Curt et al, 2004). ${ }^{5}$ The proposed simple linearization method of individual data might be of additional help for future randomized controlled trials (RCTs) to enhance comparability and reduce inclusion numbers of patients needed without changing the basis of the ASIA score. Furthermore, linear data procurement (i) increases comparability and is suitable to improve retrospective data analysis, (ii) proposes a higher efficacy to detect pharmaceutical effects and (iii) leads to a putatively reduced number of required patients. This represents an option to reduce costs performing SCI trials, thereby rendering this indication more attractive for pharmaceutical companies.

\section{Acknowledgements}

HJS is supported by the Hertie Foundation. JMS is awarded by a Poste-Rouge-Fellowship of the Centre National de la Recherche Scientific of the French Government and by the German Research Council (DFG), \#1164/1-1.

\author{
R Jaeger ${ }^{1}$, K Brechtel ${ }^{2}$, HJ Schluesener ${ }^{1}$, S Conrad $^{3}$, \\ H-P Kaps and JM Schwab ${ }^{5}$ \\ ${ }^{1}$ Institute of Brain Research, Calwer Str. 3, Tuebingen \\ University, Medical School, Germany; ${ }^{2}$ Diagnostic \\ Radiology, Hoppe-Seyler Str. 3, Tuebingen University, \\ Medical School, Germany; ${ }^{3}$ Experimental Tissue \\ Engineering, Department of Anatomy, Tuebingen \\ University, Medical School, Germany; ${ }^{4}$ Department of \\ Spinal Cord Injury, BG Trauma Center, D-72076 \\ Tuebingen, Germany; ${ }^{5}$ CNRS UMR 7102, Equipe \\ Développement Neuronal, Université Pierre et Marie \\ Curie (Paris 6), Paris, France
}

\section{References}

1 Curt A, Schwab ME, Dietz V. Providing the clinical basis for new interventional therapies: refined diagnosis and assessment of recovery after spinal cord injury. Spinal Cord 2004; 42: 1-6.

2 Clarke M, Oxman AD, Cochrane Reviewers' Handbook In: Cochrane Reviewers' Handbook [online]. Available at:. www.cochrane.dk/cochrane/handbook/handbook.htm Accessed January 62002.

3 Young W, Bracken MB. The second national acute spinal cord injury study. J Neurotrauma Suppl 1992; 9: S397-S405.

4 Geisler FH, Coleman WP, Grieco G, Poonian D. Measurements and recovery patterns in a multicenter study of acute spinal cord injury. Spine 2001; 26(Suppl): S68-S86.

5 Maynard Jr FM et al. International standards for neurological and functional classification of spinal cord Injury. American Spinal Injury Association. Spinal Cord 1997; 35: 266-274.

6 Curt A, Dietz V. Electrophysiological recordings in patients with spinal cord injury: significance for predicting outcome. Spinal Cord 1999; 37: 157-165. 